\title{
Analysis on the Situation of Water Scarcity in Beijing City Based on NARSD Model
}

\author{
Wentao Feng \\ School of Mechanical Engineering, North China Electric Power University, Baoding, 071003, China; \\ fengwentao9502@163.com
}

\section{Keywords: NARSD Model Water Scarcity}

\begin{abstract}
Water Scarcity is a problem which draws the world's attention all along. Beijing, the capital of China, is an over-exploited region when it comes to water scarcity according to the UN water scarcity map. So this paper's major concern lies in developing a model that can measure and estimate the water scarcity and then alleviate the water scarcity in Beijing city. Firstly, a NARSD Model based on the System Dynamic Model and the Nonlinear Auto-regressive Model is developed to measure the water scarcity in Beijing with the rate of water supply and water demand. Secondly, the NARSD Model is used to forecast the water situation of Beijing in 15 years from 2015 to 2030. Finally, an intervention plan is proposed to alleviate the water scarcity in Beijing city based on the sensitivity analysis of the NARSD Model. And the effectiveness of the intervention plan can be verified by making comparisons between water situation of Beijing in 15 years under intervention and water situation without intervention as is shown at the last of this paper.
\end{abstract}

\section{Introduction}

\section{- Background}

The growing scarcity of fresh and clean water is among the most important issues facing civilization in the 21 st century ${ }^{[1]}$. Water scarcity can be defined as a lack of sufficient water, or not having access to safe water supplies. $60 \%$ of the earth's population live in the water-stressed conditions, however demand for fresh and clean water are growing. There are two primary causes for water scarcity: physical scarcity and economic scarcity. Physical water scarcity, in which roughly 20 percent of the world's population now lives, occurs when there isn't enough water to meet demand. While economic scarcity occurs where water exists but poor management and lack of infrastructure limits the availability of clean water. The reason why Beijing is scarce of clean water can be summarized as two sub-reasons primarily as above: physical water scarcity and economic scarcity.

\section{The way to measure the water scarcity}

To assess the water scarcity in Beijing city, an evaluation standard should be set up firstly. Water scarcity can be described with water demand and water supply. The higher the difference between the amount of water demand (d) and the amount of water supply (s), the more serious is the water scarcity. That is, the extent of water scarcity can be described with the rate as: $\alpha=\mathrm{d} / \mathrm{s}$.

The water demand can be mainly divided into four parts: Domestic water consumption $\left(d_{1}\right)$, Water consumption for Environment $\left(d_{2}\right)$, Water consumption for Industry $\left(d_{3}\right)$ and Water consumption for Primary Industry $\left(\mathrm{d}_{4}\right)$. While the water supply is composed mainly with five main parts: Precipitation utilized quantity $\left(s_{1}\right)$, Entry water $\left(s_{2}\right)$, Reclaimed water $\left(s_{3}\right)$, Exit water $\left(s_{4}\right)$ and Sea water desalination $\left(\mathrm{s}_{5}\right)$. That is:

$$
\mathrm{d}=\mathrm{d}_{1}+\mathrm{d}_{2}+\mathrm{d}_{3}+\mathrm{d}_{4} \quad \mathrm{~s}=\mathrm{s}_{1}+\mathrm{s}_{2}+\mathrm{s}_{3}+\mathrm{s}_{4}+\mathrm{s}_{5}
$$

So the water scarcity can be described with $\alpha, d_{i}$ and $s_{i}$. 


\section{Introduction of the NARSD Model}

\section{The System Dynamic Model}

The System Dynamic Model (SDM) can not only arrange and describe the complicated connections among each element in different levels, $S=\left\{S_{i} \in S \mid i \in I\right\}$ but also deal with dynamic processes with feedback in a system ${ }^{[2]}$. The approach of SDM is decomposing the system into layers, the steps of which can be described as follows.

i. Decompose the system into several sub-systems that are connected according to the structure and layer of the system. That is, $S=\left\{S_{i} \in S \mid i \in I\right\}$.where:S is the total system, $S_{i}$ is the sub-system.

ii. Describe the sub-systems. Each sub-system consists of a basic unit and a first-order feedback loop which is composed of a state variable, a rate variable and an auxiliary variable. And the three variables can be expressed with a state equation, a rate equation and an auxiliary equation, which can be used to describe the complex changes of real system together with other variable equations, constant and mathematical equations. That is

$$
\dot{L}=P R \quad\left[\begin{array}{l}
R \\
A
\end{array}\right]=W\left[\begin{array}{l}
L \\
A
\end{array}\right]
$$

Where: $\dot{L}$ is the pure rate variable vector; $\mathrm{P}$ is the transfer matrix; $\mathrm{R}$ is the rate variable vector; A is the auxiliary variable vector; $\mathrm{W}$ is the relation matrix; $\mathrm{L}$ is the state variable vector.

The above-described mechanism can be shown Equation (1).

$$
\left\{\begin{array}{c}
d L E V /\left.d t\right|_{t}=R A T I N(t)-R A T O U T(t) \\
\operatorname{RATIN}(t)=f_{1}(L E V(t), A U X(t), E X O(t), C O N S T) \\
\operatorname{RATOUT}(t)=f_{2}(L E V(t), A U X(t), E X O(t), C O N S T) \\
\operatorname{AUX}(t)=g\left(L E V(t), A U X^{*}(t), E X O(t), C O N S T\right)
\end{array}\right.
$$

Where: LEV is the state variable; RATIN, RATOUT are the rate of input and output of the state variable; AUX, AUX* are the auxiliary variable; EXO is the exogenous variable; CONST is the constant.

However, the SDM contains a deviation which lies in its flexible in parameters determination, that is, the main parameters will keep unchanged once determined. So a model which can get over this disadvantage is supposed to be developed.

\section{- The NARSD Model improved from the System Dynamic Model}

To get over this disadvantage of the SDM, we introduce the Nonlinear Auto-regressive Model (NAR) and develop the NARSD Model. The NAR Model can be defined as follows: ${ }^{[3]}$

$$
\mathrm{y}_{\mathrm{n}}=\mathrm{G}\left(\mathrm{y}_{\mathrm{n}-1}, \ldots, \mathrm{y}_{\mathrm{n}-\mathrm{k}}, \mathrm{x}_{\mathrm{n}}, \ldots, \mathrm{x}_{\mathrm{n}-1}\right)
$$

Where: $\mathrm{x}$ is the input of model; $\mathrm{y}$ is the output of model; $\mathrm{n}$ is the discrete time; $\mathrm{G}$ is the nonlinear function.

When the data is time series, and the input of model is unknown, the expression can be rewritten as follows in an ANR form:

$$
\mathrm{y}_{\mathrm{n}}=\mathrm{F}\left(\mathrm{y}_{\mathrm{n}}, \ldots, \mathrm{y}_{\mathrm{n}}\right)+\mathrm{k} \varepsilon_{\mathrm{n}}
$$

Where: $\mathrm{F}$ is the nonlinear function; $\mathrm{k}$ is the constant, $\varepsilon_{\mathrm{n}}$ is the random variable subject to Gauss distribution.

The output of each $y$ in the NAR model is to point to the input of the neural network layer, serving as the next output of the adjustment parameters and completing the adjustment of the neural network. Therefore, the NAR model can reflect the historical information of the system, and it can be used to parameters determination in the SD Model. Hence, the NARSD Model is the combination of the NAR Model used to determine the main parameters and the SD Model used to analyze the inner correlations, which is believed to perform better in the parameters determination.

\section{Application of the NARSD Model to measure and predict the Water Scarcity in Beijing}

To application the NARSD Model, the water demand and water supply are divided into different 
sub-systems as shown in Fig.1 and Fig.2

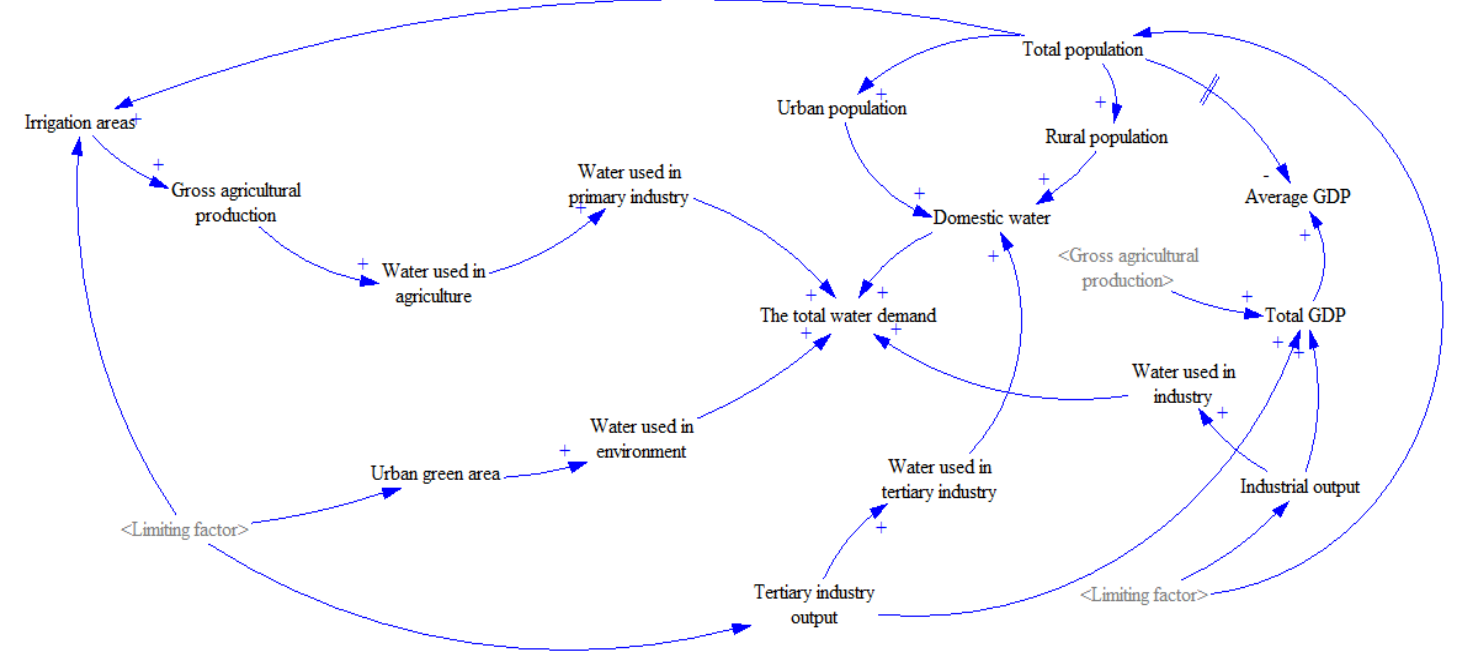

Fig.1 The sub-systems and causality diagram of water demand system

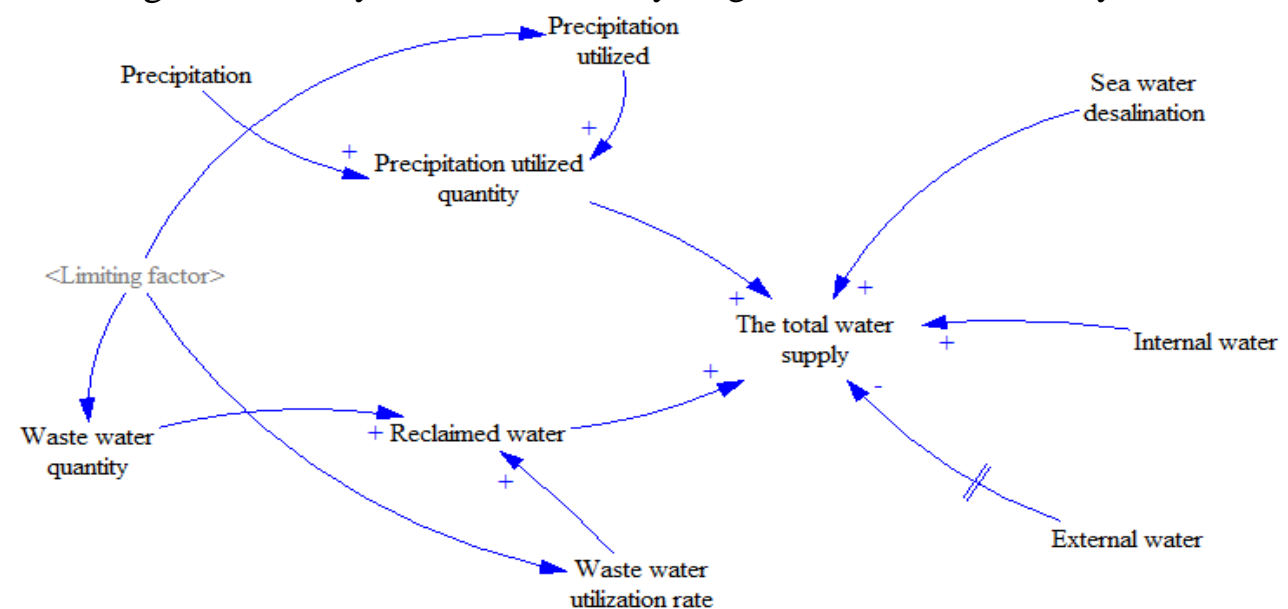

Fig. 2 The sub-systems and causality diagram of supply water system

To measure the water scarcity of Beijing in the future years, we estimate the water demand, the water supply and the rate $\alpha$ based on the NARSD model in 15 years from 2015 to 2030. And the estimation results are listed in Fig.3 and Fig.4

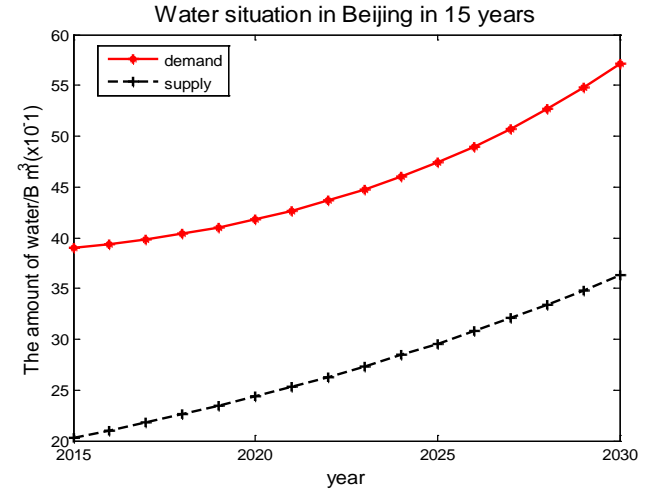

Fig.4 Water situation of Beijing in 15 years

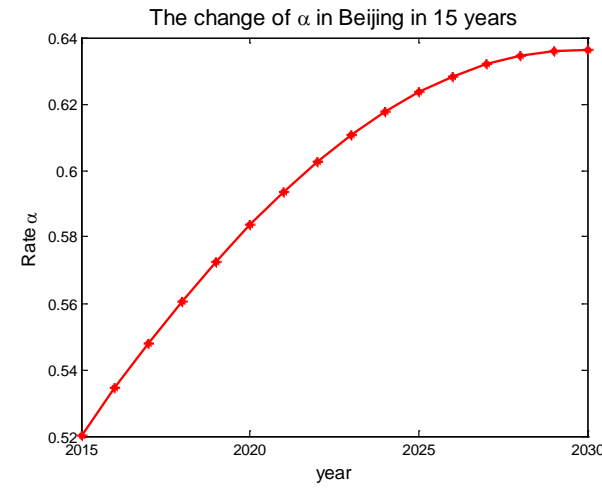

Fig.5 The change of $\alpha$ of Beijing in 15 years

\section{An intervention to alleviate water scarcity in Beijing}

As is analyzed in the previous section, interventions are supposed to be given to alleviate the shortage of water recourses more efficiently in Beijing. Here we used the measure based on the sensitivity analysis of NARSD Model. And we can learn from the results of sensitivity analysis that a minor change in several parameters can lead to a great change in the result of NARSD Model, which means a great change in the total water quantity. In other words, we can mainly adjust the 
values of these sensitive parameters to change the amount of water supply and water demand, which are supposed to be a high-efficient intervention.

Thus, our interventions take all the drivers of water scarcity into account, while mainly focus on the sensitive parameters that are AGW (water consumption per unit of gross agricultural production), COW (water consumption per unit area), POW (water consumption per capita) and $s_{2}$. In terms of water demand, the interventions can be concluded as adjusting the industrial structure and industrial optimization, improving industrial efficiency and using water-saving equipments to decrease the water consumption per unit output value of various industries by $5 \%$. Decrease other parameters such as IRR (irrigation areas growth rate), URR (urban green area growth rate) and TER (tertiary industry output growth rate) by $1 \%$. In terms of water supply, it is advised to maintain entry water and reduce exit water, at the same time, increase the value of PRR (the change rate of precipitation utilized) by $1 \%$ if necessary.

In order to show the advantage of the interventions presented in the previous section, we make a comparison of the state under interventions with the state without interventions, the value of both can calculated in the early sections. Hence, the results of them are displayed in Fig. 6 for comparison

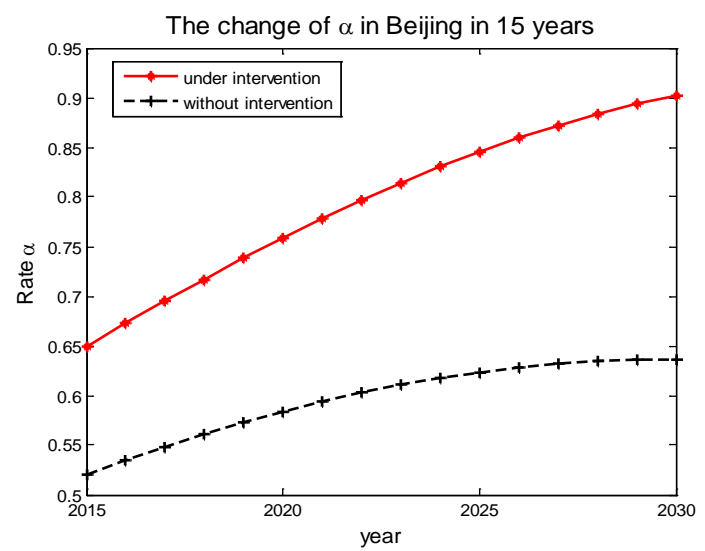

Fig.6 The change of $\alpha$ of Beijing in 15 years. Where the red line presents the rate under intervention while the black line presents the rate without intervention

In conclusion, NARSD model do well in measuring and forecasting the situation of water scarcity in Beijing and an intervention can be given to alleviate water scarcity based on the sensitivity analysis of NARSD Model. At last, this model can be applied to any other city for water scarcity analysis.

\section{References}

[1] Slobodan, P., World water dynamics: global modeling of water resources, Journal of Environment Management ,(2002)00,1-19

[2] Chunyang, H., Norio, O., Qiaofeng, Z., Peijun, S., Jingshui, Z., Modeling urban expansion scenarios by coupling cellular automata model and system dynamic model in Beijing China, Science Direct Applied Geography, 26(2006):323-345

[3] Xiang, W., Jixiang, H., Lei, S., Jing, Z., Establishment of aquaculture water prediction model based on NAR neural network, Fishery Modernization, 4(42):30-34.2015.8. 